\title{
ASPECTS PSYCHO-SOCIAUX DE LA STERILITÉ MASCULINE EN TUNISIE
}

\author{
T. Skhiri, L. Gaha et M. Nasr. \\ Centre de Santé Mentale et de Prévention - Hôpital Universitaire Fattouma Bourguiba \\ 5019 Monastir - TUNISIE
}

PSYCHO-SOCIOLOGICAL ASPECTS OF MALE INFERTILITY IN TUNISIA. A sample of 22 consultants for sterility has been studied. Sterility is badly accepted ; it is felt as a narcissic wound, keeping on a depressive mood, with loss of the spirit of initiative. The multiplication of examinations and visits to doctors is a current consequence inspite of the results of complementary examinations. The means of reparation and compensation are very much restricted. Insemination is refused in $77 \%$ of the cases. Adoption causes many cultural problems.For the majority of consultants resorting to religion results in resignation more than acceptation. Key -words: male infertility, culture.

Andrologie : 1991, 1 : 40-41

\section{INDRODUCTION :}

L'étude de la stérilité chez l'homme tunisien est un aspect nouveau dans nos traditions et dénote de la transgression d'un tabou : celui de la désacralisation de l'homme.

Lorsqu'on formule des voeux de bonheur aux jeunes mariés, on sous-entend, "faites beaucoup d'enfants et soyez heureux". La jeune épouse n'acquiert sa dimension de femme puis de mère respectée que lorsqu'elle met un enfant au monde. Quand à lhomme sa virilité est reconnue lorsque sa semence montre ses propriétés "fertilisantes".

Ainsi la grossesse et la naissance d'un enfant représentent la concrétisation de désirs et viennent également soulager les inquiétantes attentes relatives à l'angoisse de castration.La famille et la communauté sont également soulagées, les deux conjoints s'étant montré à la hauteur des espoirs mis en eux, en participant chacun selon son destin biologique à la perennité de l'espèce.

Le travail que nous avons entrepris tente de répondre à la question de l'acceptation par l'homme de sa stérilité ainsi que de ses conséquences sociales.

Présenté au $8^{\text {¿me }}$ congrès de la SALF Sousse, 6-8 septembre 1990.

\section{METHODOLOGIE DE L'ENQUETE :}

Sur le plan méthodologique, nous avons interrogé au centre de médecine appliquée, 22 personnes de sexe masculin, mariés, sauf 1 célibataire qui consultait pour se rassurer sur les conséquences éventuelles d'une ectopie testiculaire.

Nous avons utilisé un protocole comprenant les 10 items suivants :

1 - Date du mariage.

2 - Date de la première consultation.

3 - Résultats des explorations pour :

- le patient

- le conjoint

4 - Le vécu de la problématique par :

- le patient

- l'épouse

- les parents

- les beaux parents

5 - Attitude du milieu socio professionnel.

6 - Réactions du patient :

- consultations médicales

- recours à la religion

- recours aux guérisseurs.

7 - Modification du comportement

- du patient vis à vis de l'épouse

- de l'épouse vis à vis du patient.

8 - Comportement social

9 - Ațtitude vis à vis de l'adoption.

10 -Attitude vis à vis de l'insémination.

\section{RESULTATS ET DISCUSSION :}

Les consultants sont âgés de plus de 25 ans et consultent dans la majorité des cas dans l'année qui suit le mariage (tableaux 1 et 2).

Tableau 1 : Répartition en fonction de l'âge

\begin{tabular}{lcc}
\hline \multicolumn{1}{c}{ AGE } & EFFECTIFS & $\%$ \\
\hline Inférieur à 25 & 0 & 0 \\
25 à 29 ans & 7 cas & $32 \%$ \\
30 à 34 ans & 10 cas & $45 \%$ \\
35 à 39 ans & 3 cas & $14 \%$ \\
Supérieur à 40 ans & 2 cas & $9 \%$ \\
\hline TOTAL & 22 cas & $100 \%$ \\
\hline
\end{tabular}

Tableau 2 : Répartition en fonction de la durée entre le mariage et la première consultation

DUREE ENTRE MARIAGE

ET $1^{\text {ere }}$ CONSULTATION EFFECTIFS \%

\begin{tabular}{|c|c|}
\hline 1 an & 13 cas \\
\hline 1 à 2 ans & 4 cas \\
\hline 2 à 5 ans & 3 cas \\
\hline Plus que 5 ans & 2 cas \\
\hline TOTAL & $22 \mathrm{cas}$ \\
\hline
\end{tabular}

Ces 22 personnes sont originaires des régions rurales de Sousse - Kairouan et Mahdia. La plupart sont de condition socio-économique modeste, d'un niveau culturel primaire, musulmans, non pratiquants.

Les premières interrogations relatives à la grossesse ont lieu généralement au cours du ler semestre qui suit la date du mariage. La fin de la première année inaugure la période des doutes et des inquiétudes. Lorsque le doute n'est plus permis, l'entourage exhorte les époux à plus d'entrain et donnent des conseils diététiques à l'homme pour fortifier sa semence.

Après l'échec des efforts naturels, l'épouse est invitée à se faire soigner, ce qui se fait en général de bonne grâce. Cest souvent devant la négativité des examens cliniques et paracliniques, que ron se tourne vers le conjoint obéissant en cela aux conseils du médecin. Le conjoint se soumet au verdict de la médecine, non sans avoir essayé auparavant les recettes de "bonnes femmes" (miel -amandes - grains de sésame) tout en multipliant la fréquence des rapports sexuels.

Le résultat du spermogramme est attendu dans le plus grand secret par les deux conjoints, les explications du médecin conscient de l'importance de sa relation seront interprétées en termes de faiblesse séminale laissant la porte ouverte à l'espoir et à la poursuite des examens. En définitive, seul Dieu en décidera, l'homme ne peut que s'y conformer.

Lorsque les proches et amis sont mis au courant, médecine et médecins reçoivent leur lot de 
critiques, chacun rapportant d'heureux évènements survenus chez des couples condamnés par la médecine à la stérilité.

Réaction des conjoints après la révélation : Sur les 22 consultants, un seul a réagi avec une certaine désinvolture, les autres n'acceptèrent jamais leur stérilité comme étant définitive. Malgré cette réaction de déni, la plupart ont un sentiment d'échec. 1 seul évoqua la possibilité de mettre fin à ses jours ("sans enfant la vie ne vaut pas le coup d'être vécue").

En général, la faiblesse étant toujours passagère $90 \%$ des consultants conseillés par le groupe des parents et amis, vont multiplier les consultations médicales, visitent des marabouts, font des offrandes (sacrifice d'une chèvre), se soumettent aux guérisseurs. Ce n'est qu'après cette phase de lutte que la résignation s'installe laissant la volonté de Dieu s'accomplir. "Je ne peux quand même pas blasphémer en allant à l'encontre de la volonté divine", me dit l'un d'entre eux.

Au cours de notre enquête, nous n'avons pas interviewé de femmes. Nous savons par expérience que pour elles un foyer sans enfants, c'est une vie gâchée, un avenir incertain. Leur rôle de gardienne du foyer, leur besoin de maternage et d'expression de tendresse ne vont pas trouver l'appui naturel qu'est l'enfant. Un dicton populaire dit "Dieu fasse que ta maison ne soit jamais désertée".

Par l'absence d'enfant, la femme est déstabilisée dans son identité biologique et sociale, car la naissance d'un enfant marque une triple naissance, celle des deux géniteurs et celle du nouveau-né.

Dans le contexte de la stérilité masculine, la femme est d'abord soulagée, car elle n'est pas directement en cause. Elle réagit en pressant le mari culpabilisé de se faire soigner. Le secret est longtemps gardé. Seule la mère de l'époux stérile est mise au courant, le diagnostic est bien sûr rejeté, un changement de femme est souvent proposé.

Selon les dires de nos consultants les beaux parents sont censés être dans lignorance du secret, ou feignent l'indifférence. L'entourage des amis et des voisins entretiennent par leurs questions un certain agacement des époux qui banalisent, fuient les questions par de multiples astuces.

Cette attente fébrile est à notre avis, l'expression de la crainte d'un danger, danger de castration du couple et perturbation d'un ordre social et biologique. En effet, la vie sociale est organisée autour d'un certain nombre de points forts, immuables qui servent de cadre de référence. Ainsi assurer son avenir, c'est travailler, construire une maison, se marier, avoir des enfants, les élever. La voie de la mère est alors toute tracée : préserver le bon développement des enfants, leur santé, leur réussite à l'école, puis dans la société. La réaction dépressive, et le fond dépressif qui persistent tout au long de la vie du couple stérile, s'expliquent par ce manque à être et à vivre des étapes naturellement programmées.

Ces manifestations dépressives plus marquées chez la femme que chez l'homme s'expriment par une symptomatologie psycho-fonctionnelle bien spécifique. Dans notre échantillon, nous avons relevé les éléments symptomatiques suivants : perte du goût de vivre, sensation de vide, dinutilité, sentiment de tristesse ("construire une maison pour qui ?" "acheter des cadeaux pour qui?") crainte de l'avenir ("qui prendra soin de nous lorsque nous serons vieux ?")

Les fêtes : Aid, circoncision et mariages représentent une véritable catastrophe pour certains qui auront tendance à les éviter. La vie sociale et les contacts avec les autres familles se retrécissent, progressivement et finiront par donner un caractère de repli sur soi de la famille sans enfants.

Modalités de compensation : Puisqu'il faut malgré tout vivre, le recours à la religion et le respect dû à la volonté divine, aident à supporter le poids du destin.

Le divorce fut envisagé 2 fois $/ 22$ :

- par une jeune épouse de 18 ans.

- proposé comme solution par un des consultants à son épouse qui a refusé.

Les 20 restants semblent avoir accepté leur sort, non sans agressivité et angoisse.

L'espoir n'étant jamais tout à fait perdu, la dynamique du couple se réorganise. L'homme exprime sa tendresse et sa sollicitude à son épouse alors que cete dernière en marque de maternage, le désigne comme étant aussi son enfant. Ceci fut exprimé explicitement par un des consultants.

L'adoption : pose des problèmes culturels et religieux, elle est choisie comme solution 3 fois/22. 11 couples n'avaient pas opté ou réfléchi à cette question.

Parmi ceux qui sont pour, ce choix se porte préférentiellement pour un membre de la famille (neveu ou nièce). Il y a lieu de souligner par ailleurs que l'adoption est régie par la loi Tunisienne (Loi $N^{\circ} 58$ - 27 du 24 mars 1958) qui fixe: 1) Les conditions requises pour adopter un enfant. - L'adoptant doit être en effet majeur de l'un ou de l'autre sexe, marié et jouissant de la pleine capacité civile.

- Il doit être également de bonne moralité, sain de corps et d'esprit et en mesure de subvenir aux besoins de l'adopté.

2) Les conditions relatives à l'adopté qui doit être un enfant mineur de l'un ou de l'autre sexe.

3) L'acte d'adoption qui est établi par un jugement rendu en présence de l'adoptant et de son conjoint dont le consentement est nécessaire.

Le jugement rendu est définitif et l'adopté a les mêmes droits et les mêmes obligations que la loi reconnait et impose aux enfants légitimes.

L'insémination : - Pour : 5

Contre : 17

- Ceux qui sont contre, expriment leur refus farouche, considérant l'insémination comme l'équivalent d'un don de sa propre femme.

- Cependant, certains consultants ont accepté l'insémintation lorsqu'il s'agit de leur propre sperme.

\section{ACCEPTATION DE LA STERILITE :} APERÇU PSYCHO-DYNAMIQUE

Pour nous résumer, la dynamique conjugale relationnelle et sociale d'un couple stérile passe par 4 phases :

1) Prise de conscience de la réalité avec réaction anxieuse et dépressive

2) Phase active de réparation :

-conseils diététiques.

-maraboutisme (sacrifices).

-recours aux guérisseurs.

-aux médecins.

3) Phase de découragement, relation conflictuelle entre les époux soutenus par leurs familles respectives.

4) Cette $3^{\text {zme }}$ phase débouche sur la $4^{\text {eme }}$ soit :

- divorce ou

- acceptation d'une vie familiale sans enfant dans un équilibre précaire avec repli du couple sur soi et régression, les deux conjoints restant dans un statut de jeunes. La capacité de compensation du manque est fonction de la personne, de son niveau socio-culturel.

Souvent la femme se déprécie à ses propres yeux et se réfugie dans la maladie. Quant à l'homme dont la vie est orientée vers l'extérieur, le surinvestissement de la vie professionnelle est un recours fréquent.

\section{CONCLUSION}

L'échantillon que nous avons étudié n'est pas exhaustif et ne donne qu'une idée partielle et subjective des problèmes liés à la stérilité masculine. En effet, ni les conjointes, ni les parents, ni les beaux parents ne figurent dans notre échantillon.

Néanmoins, on peut dire que cet état de stérilité est vécu comme un manque douloureusement ressenti, une blessure profonde qui bouleverse l'investissement familial et social de l'homme stérile. Les modalités compensatoires sont assez pauvres, le recours au destin et à la religion reste le meilleur moyen de défense pour l'équilibre individuel et social.

Tous ces facteurs invoqués font dégager la nécessité d'une aide psycho-sociale au couple stérile. 